\title{
Development of a Radio Enabler for Reconfiguration Management within the IEEE P1900.4 Working Group
}

\author{
Oliver Holland ${ }^{1}$, Markus Muck ${ }^{2}$, Patricia Martigne ${ }^{3}$, Didier Bourse ${ }^{2}$, Pascal Cordier ${ }^{3}$, Sana Ben \\ Jemaa $^{3}$, Paul Houze ${ }^{3}$, David Grandblaise ${ }^{2}$, Clemens Klöck ${ }^{4}$, Tobias Renk ${ }^{4}$, Jianming Pan ${ }^{5}$, Peter \\ Slanina $^{5}$, Klaus Mößner ${ }^{6}$, Lorenza Giupponi ${ }^{7}$, Jordi Pérez Romero ${ }^{7}$, Ramón Agusti ${ }^{7}$, Alireza Attar ${ }^{1}$, A. \\ Hamid Aghvami ${ }^{1}$
}

\begin{abstract}
An important emerging capability is for mobile terminals to be dynamically reconfigured. Through ongoing advances in technology such as software defined radio, reconfiguration of mobile terminals will in the near future be achievable across all layers of the protocol stack. However, along with the capability for such wide-ranging reconfiguration comes the need to manage reconfiguration procedures. This is necessary to coordinate reconfigurations, to ensure that there are no negative effects (e.g. interference to other RATs) as a result of reconfigurations, and to leverage maximal potential benefits of reconfiguration and ensuing technologies such as those involving dynamic spectrum access. The IEEE P1900.4 working group is therefore defining three building blocks for reconfiguration management: Network Reconfiguration Management (NRM), Terminal Reconfiguration Management (TRM), and a radio enabler to provide connectivity between the NRM and TRMs. In this paper we concentrate on aspects of the radio enabler, highlighting its relevance in heterogeneous radio access scenarios, its advantages, and some aspects of its technical realization.
\end{abstract}

Index Terms-Reconfiguration, Radio Resource Optimization

\section{INTRODUCTION}

$\mathrm{R}$ ECONFIGURATION management is a complex process, requiring a plethora of information to be transferred among the system(s) and mobile devices involved in reconfiguration procedures [1]. The same applies to the management of technologies involving dynamic spectrum access which are facilitated by reconfiguration, such as cognitive radio [2][3] and policy-based adaptive radio [3]. In many cases, such information must be presented among

\footnotetext{
${ }^{1}$ King's College London, London, UK. \{oliver.holland, ali.attar, hamid.aghvami\}@kcl.ac.uk

${ }^{2}$ Motorola Labs, Paris, France. \{markus.muck, didier.bourse, david.grandblaise\}@motorola.com

${ }^{3}$ France Telecom R\&D, Paris, France. \{patricia.martigne, pascal.cordier, sana.benjemaa, paul.houze\}@ orange-ftgroup.com

${ }^{4}$ University of Karlsruhe, Karlsruhe, Germany. \{kloeck, renk\}@int.unikarlsruhe.de

${ }^{5}$ Siemens Austria, Vienna, Austria. \{jianming.pan, peter.slanina\}@siemens.com

${ }^{6}$ University of Surrey, Guildford, Surrey, UK. k.moessner@surrey.ac.uk

${ }^{7}$ University Polytechnic of Cataluna (UPC), Barcelona, Spain. \{lorenza.giupponi, jorperez, ramon\}@tsc.upc.edu
}

devices and network elements in a coordinated and complete fashion, so that an informed decision can be made on a reconfiguration or associated change without the risk of acting on false positives or inconclusive data.

Currently among heterogeneous wireless networks, there is no standardized means for reconfiguration management related information to be transferred between different networks and terminals. In this paper, we therefore introduce the concept of a radio enabler for reconfiguration management, as envisaged within the IEEE P1900.4 working group [4]. All devices can listen to and likely transmit upon this radio enabler in order to obtain/convey reconfiguration management and associated information in as efficient a manner as possible.

Among the many uses for this envisaged radio enabler, devices might receive context information (including, for example, information about connectivity options upon startup) far more efficiently, just by listening to a single channel instead of having to scan among multiple network pilots. As other benefits, reconfiguration processes can be coordinated among affected elements, the transferal of policies/directives and decisions related to a reconfiguration can be expedited or indeed made possible, and dynamic spectrum allocation technologies ensuing from reconfiguration, such as cognitive radio, can be facilitated through knowledge transferal (e.g. about spectrum usage) among heterogeneous devices and network elements. To summarize, we see this radio enabler as being an essential component for future reconfigurable devices and network elements, and as being an important facilitator for dynamic spectrum access procedures both through assisting reconfiguration processes and through benefiting associated technologies such as cognitive radio.

This paper is structured as follows. In the following section the scene is set through introduction of the IEEE P1900.4 Working Group and the context of the radio enabler building block within this Working Group. In section 3, specifics on the radio enabler are conveyed, including its basic structure through specification in start-up and ongoing information phases. Continuing the detail on the radio enabler, section 4 highlights some constraints on it, and by necessity also some of its proposed technical characteristics. After proposing some 


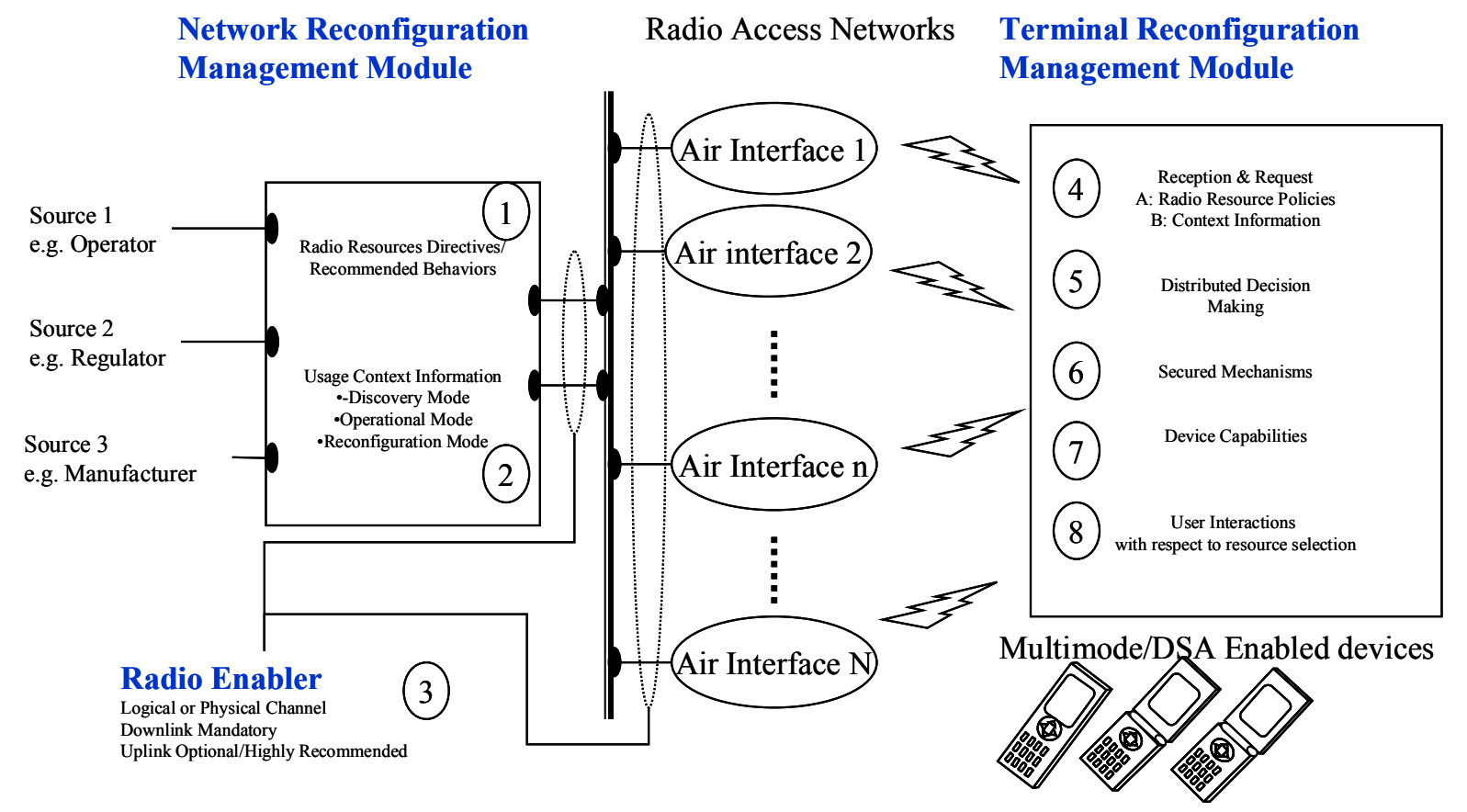

Figure 1: Overall plan of architectural elements envisaged in IEEE P1900.4

specific envisaged use-cases for the radio enabler related to dynamic spectrum access in section 5 , the paper concludes in section 6 .

\section{THE IEEE P1900.4 WORKING GROUP}

The purpose of the IEEE P1900.4 Working Group is to define architectural network and device elements and inherent information exchange between these elements, including information model representation, in order to facilitate reconfiguration management and the optimization of radio resource usage. This will lead to an improvement in overall capacity, spectral efficiency, quality of service, and device utility. Distributed Decision Making (DDM) functionalities, corresponding context and constraints information and procedures, are essential for this work. It is intended for aspects of DDM to be addressed at a later stage.

Within the context of this objective, Figure 1 illustrates the elements envisaged within P1900.4 for reconfiguration management: The Network Reconfiguration Management (NRM) module, Terminal Reconfiguration Management (TRM) modules, and the Radio Enabler for reconfiguration management. It is expected for there will be a single NRM module (or maybe a handful of NRMs) across networks, which is/are able to deal with the functionalities highlighted in Figure 1, such as provision of directive/recommended behaviors to terminals dependent on their current context, as well as the provision of context information related to connectivity discovery, ongoing operation and optimization (e.g. changing context to satisfy QoS constraints), and reconfiguration possibilities. Several sources may provide information to the NRM modules, including operators, regulators, and manufacturers. The NRM will collect and operate on this information in order to derive precise guidance, such as contexts, policies and directives, to convey to terminals such that they know exactly the bounds within which they may operate, as well as the opportunities that are open to them.

A TRM module will exist in each terminal, and will take inputs from the terminal such as that related to the terminal's capabilities as well as observed radio link quality and QoS, in order to assist in information provision such that networks can decide upon a reconfiguration and/or associated functionality such as a radio resource optimization, or the nature of constraints that will be conveyed to terminals within which they must operate. The radio enabler part simply provides the link between the NRM and TRMs, where it is important to note that this radio enabler must operate over many different possible air interfaces (air interfaces 1..n in Figure 1) and will likely be assisted by bearers of the networks that the terminals are already using. Ultimately, it will also be partly provided over a means such as a harmonized broadcast channel/RAT for devices to listen to (air interface $\mathrm{N}$ in Figure 1). It is under further investigation whether this channel $\mathrm{N}$ should be a logical channel or a novel physical channel.

The P1900.4 Working Group has identified two distinct operational phases for which the radio enabler will have a different role: (a) the start-up phase, where a terminal is being switched on or is in a new environment, and needs to learn about available RATs in an efficient manner, and (b) the ongoing phase, where the terminal is already in working mode facing varying conditions, affecting e.g. QoS and radio resource availabilities, thus presenting various benefits/opportunities for reconfiguration of the RAT with which it is associated. 


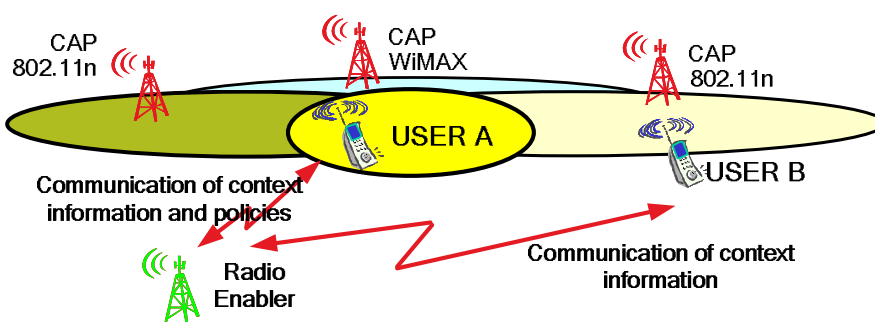

(a)

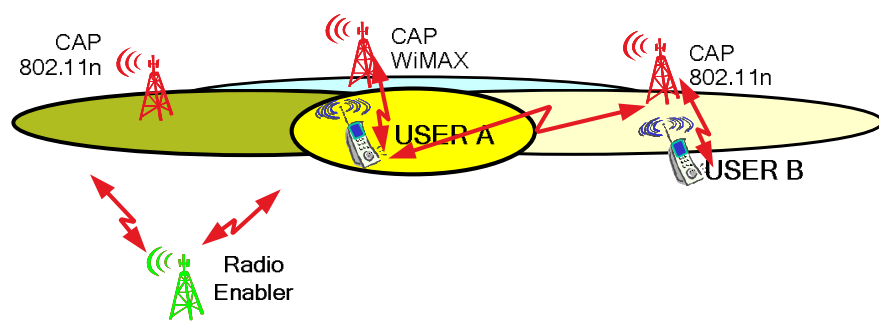

(c)

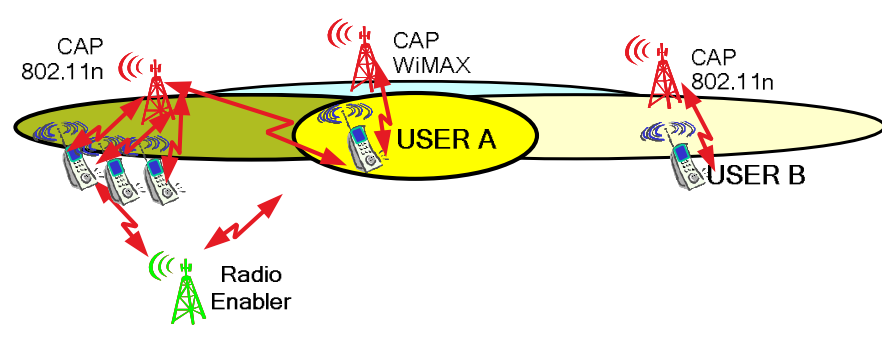

(e)

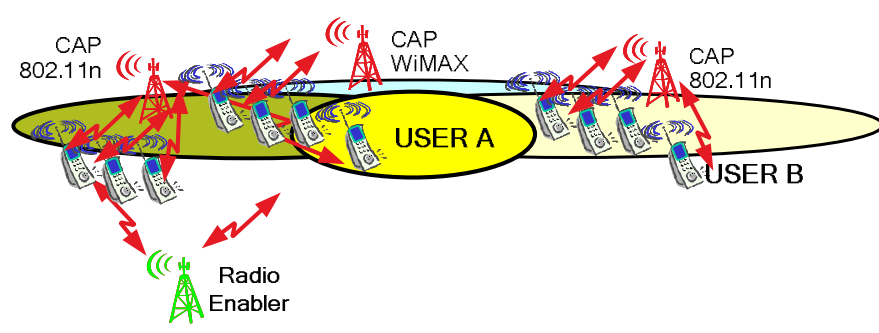

(g)

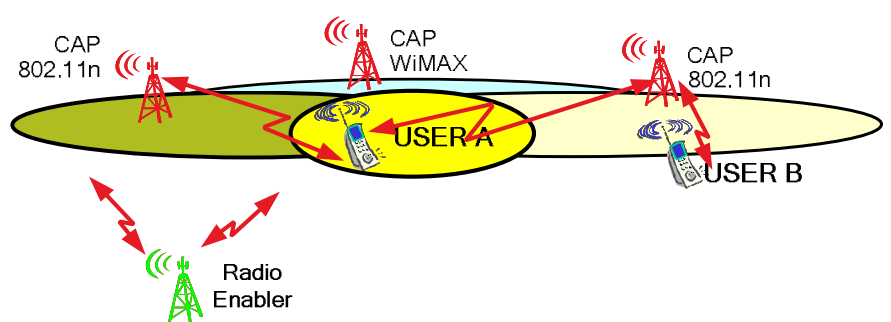

(b)

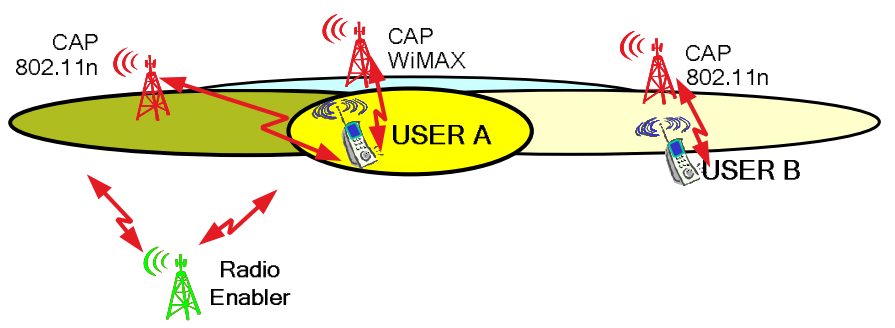

(d)

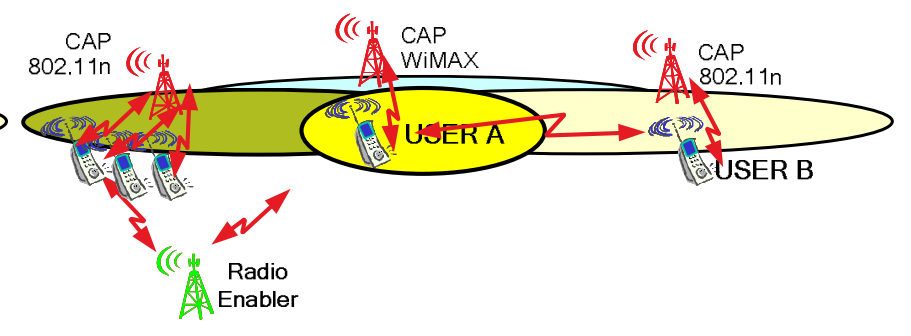

(f)

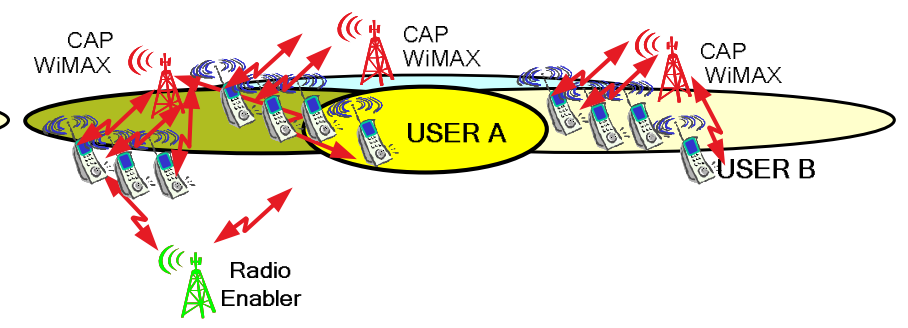

(h)

Figure 2: Storyline sequence of events emphasizing the context of the radio enabler within the P1900.4 working group

\section{A. Scenarios for Reconfiguration Management}

Pertinent to the scope of P1900.4, the following scenarios for reconfiguration management are envisaged. These are chosen as they cover the vast majority of eventualities which might lead to reconfiguration and/or associated resource reallocation being required:

- Scenario 1: The network context changes, and users adapt to optimally use available resources, e.g., an operator adds/removes RATs (the base station is no longer operational, etc.),

- Scenario 2: The user context changes and remaining users reconfigure/adapt to optimally use available resources, e.g., users arrive/leave an area,

- Scenario 3: The user behavior changes and RATs on network side are reconfigured to a different standard, e.g., the network switches from WiMAX [5] to IEEE802.11n [6] if a large number of users are suddenly close to the base station.

To align these scenarios with a real-world situation, while emphasizing the significance of the radio enabler, they are highlighted within the storyline sequence of events depicted in Figure 2:

1. Figure 2(a): User ' $A$ ' switches on and looks for available RATs (start-up context information is obtained via the radio enabler),

2. Figure 2(b): User 'A' chooses its services autonomously: It targets a low-latency VoIP communication means in parallel with a high datarate video streaming application, provided by the 


\begin{tabular}{|l|l|l|}
\hline Characteristic & Start-up Phase & Ongoing Phase \\
\hline Information conveyed & $\begin{array}{l}\text { Start-up information, e.g. context } \\
\text { information on available networks at that } \\
\text { location }\end{array}$ & $\begin{array}{l}\text { Ongoing information, e.g. much } \\
\text { more detailed context information, } \\
\text { policies for reconfiguration } \\
\text { management, feedback } \\
\text { measurements, etc. }\end{array}$ \\
\hline Channel bitrate & $\begin{array}{l}\text { Relatively low bitrate per location area. } \\
\text { However, channel bitrate must be } \\
\text { relatively high in general to cover a large } \\
\text { number of location areas and to cover a } \\
\text { large broadcast area }\end{array}$ & $\begin{array}{l}\text { Relatively high bitrate, dependent } \\
\text { on the bearer being used }\end{array}$ \\
\hline Data direction & $\begin{array}{l}\text { Downlink. Probably does not include } \\
\text { uplink, although this is under further } \\
\text { investigation }\end{array}$ & $\begin{array}{l}\text { Downlink. Almost certainly } \\
\text { includes uplink as well }\end{array}$ \\
\hline Bearer & $\begin{array}{l}\text { Most likely a harmonised frequency } \\
\text { band, wide-area coverage. Might be a } \\
\text { novel RAT, or a legacy technology of } \\
\text { appropriate characteristics (to be } \\
\text { decided) }\end{array}$ & $\begin{array}{l}\text { A bearer in a operator's network } \\
\text { (e.g. a UMTS bearer) }\end{array}$ \\
\hline
\end{tabular}

Table 1: Start-up and ongoing phases of the radio enabler, and associated characteristics

IEEE802.11n networks. This choice is made through analyzing start-up information obtained via the radio enabler,

3. Figure 2(c): At a later stage, user ' $A$ ', upon analyzing its QoS, finds it insufficient. New parameters are chosen autonomously, resulting in user ' $A$ ' connecting to the WiMAX and IEEE802.11n networks concurrently to receive its services. This change is made through analyzing ongoing information obtained via the radio enabler,

4. Figure 2(d): User ' $A$ ' further adapts its parameter sets autonomously, targeting a better usage of resources. The new strategy, where an alternative IEEE802.11n network is used, turns out to be optimum. Again, this change is made through analyzing ongoing information obtained via the radio enabler,

5. Figure 2(e): Suddenly, new users arrive (user context changes) and the throughput of the IEEE802.11n connection of User 'A' decreases,

6. Figure 2(f): The communication parameters are again adapted autonomously by User ' $A$ ', and it connects to the other IEEE802.11n network,

7. Figure 2(g): Even more users arrive. In order to optimize network behavior, the network decides to change the communication rules: the number of connections is limited to one per user. This change in policy is communicated over the radio enabler, and users adapt to this new constraint,

8. Figure 2(h): Several users linked to the IEEE802.11n access points (APs) move away from the AP locations. As facilitated by the radio enabler, the network decides to reconfigure the AP technologies from IEEE802.11n to IEEE802.16 in order to provide wider coverage and maintain the connections (the network context changes).

\section{THE RADIO ENABLER BLOCK FOR RECONFIGURATION MANAGEMENT}

Having introduced the scope and context of the radio enabler block, this section describes some of its possible characteristics in a technical realization.

\section{A. Structure of the Radio Enabler}

The sub-building blocks in Figure 3 are intended to provide a basis for future work on the radio enabler within P1900.4. The upper block, "Mapping of information (protocols) on to data transport channels," corresponds to using e.g. the information capabilities in an operator's existing network to convey the enabler. The lower block, "Mapping of information (protocols) on to a channel dedicated to protocol information exchange," corresponds to the introduction of a novel harmonized band for the radio enabler, either using a current RAT or perhaps a novel one created for this purpose.

The radio enabler is anticipated to be conveyed in two parts: that for start-up information, and that for ongoing information. The start-up information, in most cases, corresponds to the lower block in Figure 3, whereas the ongoing information corresponds to the upper one. Further detail on some special cases affecting these assumptions may nevertheless be inferred from the following paragraphs.

\section{Mapping of information (protocols) on to data transport channels. \\ Mapping of information (protocols) on to a channel dedicated to protocol information exchange.}

Figure 3: Sub-building blocks of the radio enabler 


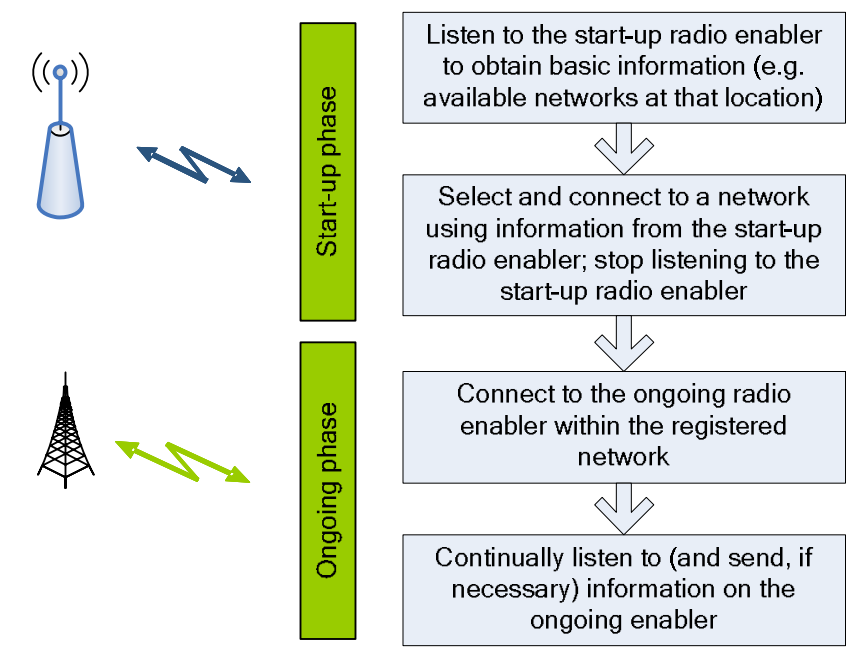

Figure 4: Start-up process for a terminal using the radio enabler

The expected characteristics of the start-up and ongoing parts of the enabler are indicated in some detail in Table 1. Under a significant proposal being considered for possible implementation, the start-up information provision is defined as being a wide-area coverage resource which either uses a new radio interface, or perhaps uses a legacy technology of appropriate characteristics (e.g. possibly DVB). In both cases it is anticipated for the start-up part to convey information on some form of harmonized band, although importantly, this part is not provided by bearers from a frequency band of an operator's legacy system - for this reason it is often referred to as "out-band". Information from the start-up part may, however, be copied to the operators' dedicated channels for the purpose of terminals listening to the radio enabler on a last-registered network, without having to switch to the P1900.4 dedicated (harmonized) band every time they power up. Moreover, in some distinct cases it might be feasible to also mirror such start-up information through other means, for example through a location-specific logical channel using an existing Internet connection. Such solutions will further enhance the scope of the enabler.

All terminals can immediately listen to the start-up part at any instance necessary to obtain vital information, where this information is anticipated to be location-dependent. It is expected for this part to cyclically broadcast start-up information to devices (e.g. available networks at that location), although due to it being on a different frequency/RAT from the other bearers the device will use, it is unlikely that it will be used for ongoing reconfiguration management information. Hence once the start-up information is obtained, it is anticipated that the device will switch to the ongoing part, and in this process will discontinue reception of the start-up part in order to avoid the need to listen to more than one frequency/RAT alternately or concurrently.

The ongoing part is anticipated to most-likely be provided by a bearer of an operator's network, and may simply be a logical link. Different devices that are registered with different networks therefore utilize different bearers for the ongoing part. The ongoing part is obtained by a device first listening to

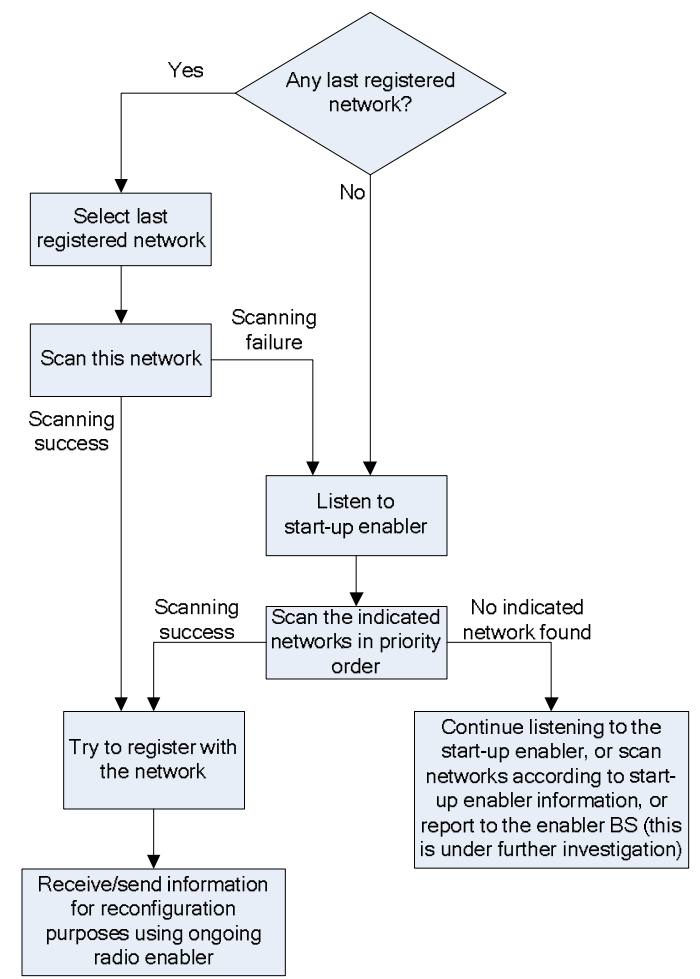

Figure 5: State diagram for network selection using the radio enabler

the start-up part then switching to the ongoing part within a network it registers with, as selected from information about networks at that location specified in the start-up part. The ongoing part is expected to provide a higher bitrate than the start-up part, and importantly is much more likely to provide uplink as well as downlink information transfer. As well as conveying operational information to/from terminals, the ongoing part might also periodically transmit the information that is given by the start-up part. This is so that terminals may stay camped on the ongoing part upon having a registered (or last-registered) network, instead of having to periodically switch (or concurrently listen to) the start-up part to ensure obtaining the complete information set.

Through this provision of start-up and ongoing radio enabler parts, the process a terminal will follow upon powerup is as in Figure 4. Moreover, the state diagram for network selection, including the important possibility of terminals maintaining information on last-registered networks, is as in Figure 5.

\section{TeChNOLOGicAl CONSTRAINTS ON, AND CHARACTERISTICS OF, THE RADIO ENABLER}

In this section, the technological constraints on the radio enabler, and by necessity some of its further characteristics, are explained.

\section{A. Start-up Radio Enabler: Location Resolution vs. Complexity of Start-up Information and Data Rate}

First, a particular technical constraint related to the start-up radio enabler is considered. Here it is necessary to detail one 
considered approach to the start-up radio enabler, as depicted in Figure 6. Information for a range of 'meshes' (corresponding to discrete coordinate areas) is broadcast to a wide area, and terminals extract the local list of existing radio systems available per operator, for their 'mesh' - see e.g. [7],[8]. For each network operator, the preferred technologies are indicated as paired with the preferred frequencies to camp on at start-up of the terminal. Hence through listening to the start-up radio enabler the terminal extracts relevant information related to its geographical position, where its geographical position is known in advance by means of a geolocation technology. The terminal then proceeds to select a network accordingly.

The formatting of data on the start-up radio enabler can be based on the assumption that a relatively limited number of technologies and networks are available within the radio enabler broadcast area. Hence each 'mesh' in this area will just need to point to a list of "connection configurations," updated by each operator as depicted in the lower half of Figure 6. There is a trade-off of the amount of information that can be conveyed in each 'mesh', with the size of 'meshes'. In assessing this trade-off based on the aforementioned approach, and using Figure 7 for reference, the total start-up radio enabler content size (per period) is determined by $n b$ Conf.ConfSize+nbMeshes. (LocSize+nbConf). Noting that the total number of 'meshes' nbMeshes is simply the broadcast area for the start-up radio enabler divided by the area of each 'mesh', thence performing calculations to the above effect, it becomes clear that the scalability of this approach is appropriate for the representation of start-up information.

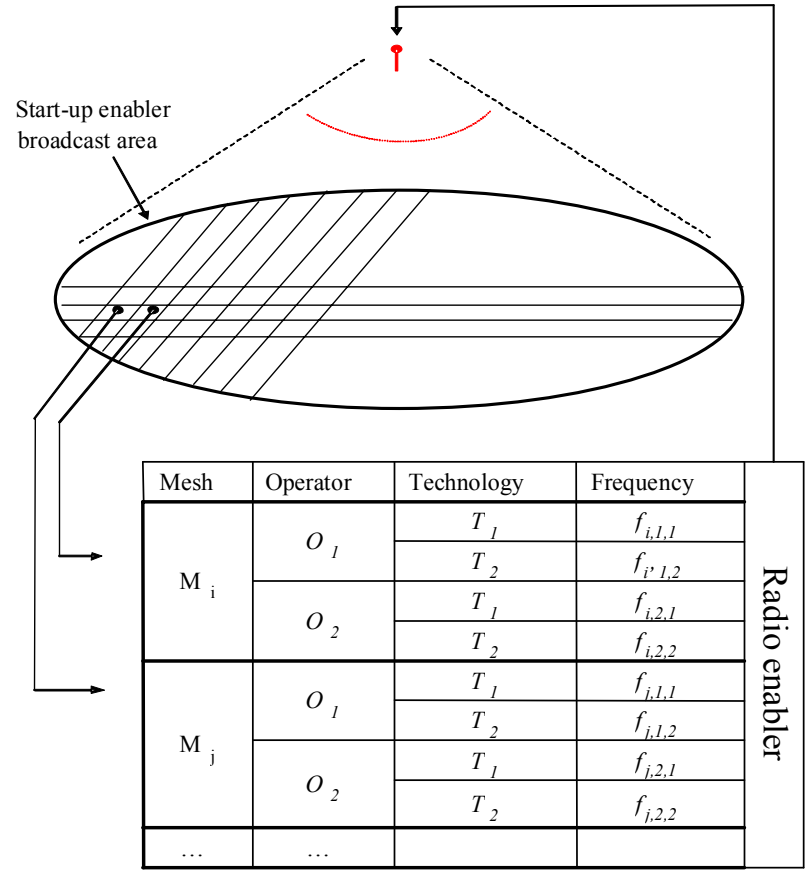

Figure 6: A considered approach for the start-up radio enabler

\section{B. Start-up Radio Enabler: Data Reliability, Dependability, and Error Rate}

An important constraint on the start-up radio enabler is the need for data reliability. In many scenarios, it is essential for receivers to know that the information they have received has not been corrupted by the channel, such that they do not then act based on incorrect information. Some form of error checking is therefore necessary on the start-up radio enabler, hence a checksum attributed to each chunk of data must be transmitted on the start-up enabler, along with that data. Given the use of such a checksum, there is still a small probability

\section{Geo-location information size: LocSize}

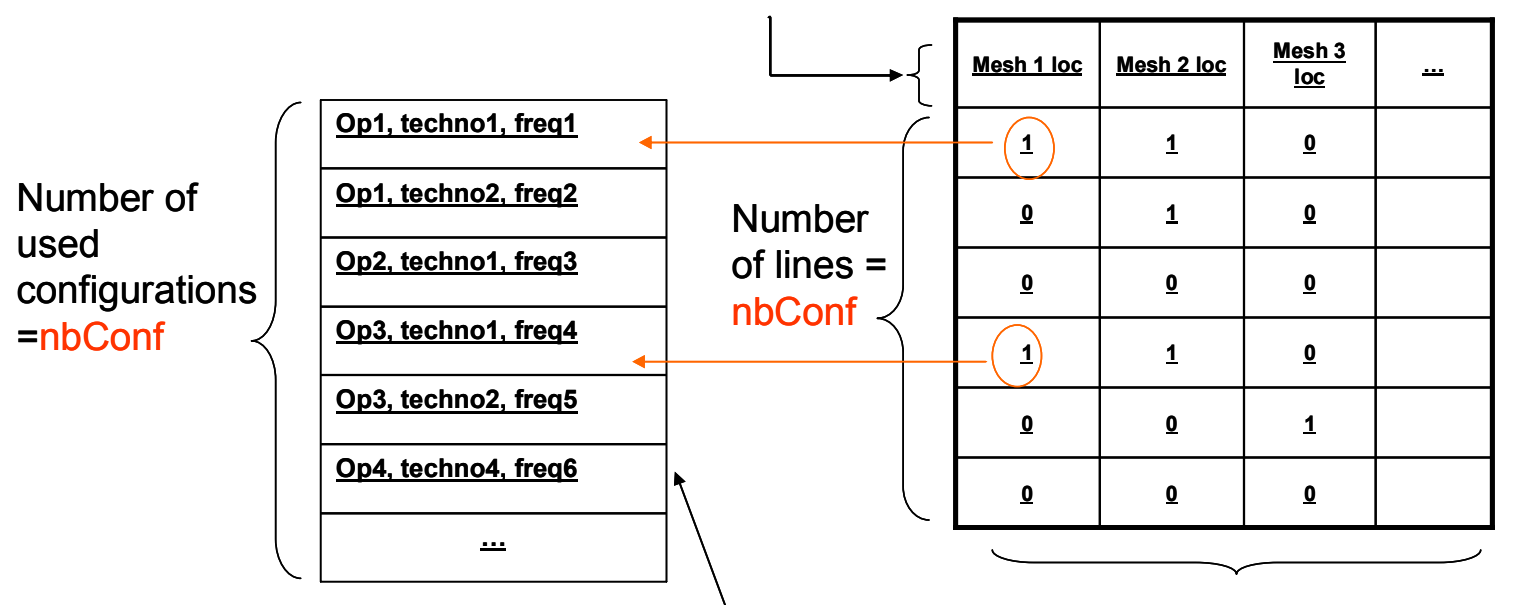

Triplet (op,techno,freq) information size: ConfSize

Number of meshes: nbMeshes

Figure 7: Information-per-period constraint on the out-band radio enabler 
that checksum calculations might give the same result for different input arguments (i.e. blocks of data). In the light of this, a reliability constraint on the radio enabler should specify a probability that the received information is correct given a verified checksum.

In a similar vein to the above, dependability of information is also a concern for the start-up enabler. In this context, a constraint here might be a certain "level of trust" being attributed to the information. In order to improve security in this context, a cryptographic hash function to generate a checksum could provide a better solution than a cyclic redundancy check, due to the one-way property of cryptographic hash functions. Intensive further work on such security for the start-up enabler however is needed.

Somewhat related to the reliability and dependability of information on the start-up enabler, another constraint might be a specified maximum error rate for the utilized information bearer, so as to minimize corruption of the conveyed information and to "nip the problem in the bud". Means to reduce this error rate include the use of forward error correction in conjunction with interleaving, among others. The exact block size over which redundancy bits are calculated could be considered as a constraint on the radio enabler in this context, where relevant factors affecting this constraint include the data overhead that the "coding rate" implies for the link, the latency involved in waiting for data to arrive for encoding/decoding, and the coding processing requirement, among others. Returning to error detection in assessment of the block size constraint over which such a calculation is done, relevant factors in this case include minimization of the probability of data being unnecessarily discarded (due to being in the same block as erroneous bits), the data/processing overhead of the error detection calculation (these are in fact likely to be small in most cases), and the way information is represented on the radio enabler. For instance, there is no logic in performing error detection on a per-'mesh' basis (see Figure 6 ), if the exact size of the 'mesh' or the definition of the exact information for the 'mesh' is represented by some kind of inheritance from other 'meshes' or other parts of the radio enabler data period.

Note that as regards all of the above constraints on the radio enabler, for many legacy systems forward error correction and data reliability/dependability will already be provided for according to the specification of the legacy system. Hence for the purpose of conveying the start-up radio enabler using a legacy system (e.g. DVB), it is simply a matter of matching the start-up enabler requirements to those of the utilized system (logical channel), and bridging the gap. Given the creation of a novel radio interface to convey the start-up enabler however, all of the above constraints must be incorporated into the design of that radio interface.

\section{Ongoing Radio Enabler}

Constraints on and technological characteristics of the ongoing radio enabler in some respects match those of the start-up enabler. For example, the "deltas" between the legacy technologies being used for the ongoing enabler and requirements for the enabler in term of reliability, dependability, error rate etc, must be bridged using higherlevel mechanisms. It is likely that a small proportion of the information for the ongoing radio enabler must be the same for all ongoing enablers (in different networks) at that location. A prime example of this is the need for the ongoing radio enabler to always convey all of the start-up information in addition to the ongoing information exchange. Hence it is anticipated that, as well as other constraints, there will be a common minimum acceptable data rate required of the ongoing radio enablers, despite them being provided by different networks.

Another constraint/technical characteristic on the ongoing radio enabler is the need for an uplink. Whereas an uplink for the start-up radio enabler will likely not be provided, for the ongoing enabler an uplink is useful and is likely necessary to convey various information to the NRM. Such information might include, for example, reports on the QoS that terminals are experiencing in order for the NRM to initiate an appropriate reconfiguration/optimization based on maximizing the collective QoS for the system(s) in question.

\section{THE RADIO ENABLER AS A FACILITATOR FOR SECONDARY SPECTRUM USAGE}

Within its wide range of possible applications, the radio enabler facilitates the secondary usage of licensed radio spectrum bands that are temporarily or locally unused, such that more efficient spectrum occupancy may follow. There is a quite unexplored secondary market that could exploit such unused frequency bands, in both spatial and time domains. Some example cases in which such spectrum could be exploited are, e.g., wireless infrastructureless systems with low cost receivers, communication of devices in personal area networks, etc. In these scenarios, the radio enabler is not only able to serve the cellular market but it also plays a key role in discovering unused frequency bands for cognitive radios.

Focusing on the typical reconfigurable cognitive radio, the requirement for safe secondary usage is first to discover the unused bands, then to take advantage of them provided that they do not interfere or jam the primary market spectrum users. In this sense, there is a set of both technical and economical challenges to be faced to ensure feasible coexistence of primary and secondary markets. These are detailed as follows.

1) The Radio Enabler Facilitating Fast Identification of

Unused Frequency Bands.

For a reconfigurable terminal to operate in the secondary market, it should first detect spectrum holes. Any secondary receiver attempting to scan the whole spectrum (which might have a range of several $\mathrm{GHz}$ ) to look for holes, would be very complex and would require a considerable scanning time, leading to it being a high-cost device. In such a scenario, the use of a radio enabler to indicate, for each geographical region (i.e. each 'mesh'), the frequencies available for secondary use, would be extremely helpful to terminals. 


\section{2) The Radio Enabler Facilitating Interference-Free} Operation of the Secondary Market

The other users affected by this process, particularly primary users, must trust in the satisfactory operation of the process such that they do not experience interference and their QoSs are not degraded. Furthermore, primary users must have preference in the usage of spectrum, with respect to secondary users, but at the same time some minimum bandwidth for link maintenance of the secondary users would be desirable. In this context, the radio enabler might avoid interference from secondary users, because any secondary terminal would know after radio enabler detection the specific frequencies that are being used in the mesh, even if it is not able to detect them due to, e.g., shadowing. Therefore, the hidden terminal effect can be avoided by the radio enabler. As another possibility, operators owning spectrum bands could help in managing the interference of secondary users by maintaining a precise database for propagation prediction and interference, dependent on the specific network planning they have at each specific time and region. From this database, the information on frequencies available for secondary usage could easily be fed into the radio enabler.

\section{3) The Radio Enabler Facilitating a Proper Business \\ Model for the Secondary Market}

A non-regulated secondary use of spectrum, in which terminals autonomously decide the frequencies upon which they can transmit, would not financially benefit the spectrum owners (e.g. operators). Hence operators might be reluctant to allow their bands to be secondary-used. However, the radio enabler provides a regulated framework for secondary usage, in the sense that owners of temporarily released frequency bands can be clearly identified by the spectrum regulator, such that the regulator can economically promote the release of frequency bands for the sake of improved spectrum usage. Moreover, stringent controls on terminals accessing the radio enabler for secondary usage could be enforced, to avoid interference issues. Consequently, cellular operators could actually benefit from the secondary usage of their spectrum through the radio enabler.

\section{4) The Radio Enabler Facilitating Secondary Market \\ Dynamic Spectrum Allocation}

Subsections (1) to (3) have pointed out the need for a radio enabler allowing opportunistic secondary system usage (with cognitive radio capabilities, at the terminal side). Another secondary spectrum usage scenario, albeit in a slightly different context from the described enabler in previous sections, consists in two or more cellular networks, where each operator can temporally rent out dynamically some spectrum on a frequency carrier basis to some secondary neighboring cells. Depending on the spectrum need over the time of the day, each BS can act as a primary or secondary system. The renting out or renting in related procedures between the primary and secondary BSs can be handled by over-the-air communications between BSs. These procedures can be supported through a radio enabler. In that case of application, from the primary BS perspective, the radio enabler could support information related to spectrum auction advertisement, discovery, information related to negotiation process, radio resources scheduling information to allow several secondary BSs to enter simultaneously in communication with the primary BS, etc. On the other hand, from the secondary BS perspective, the radio enabler could be a medium allowing the secondary BS to make a request for temporally spectrum demand. Primary BS could hear this message and come into communications with the secondary BS if this primary BS is ready to rent out some spectrum. Regardless of the case aforementioned, this type of radio enabler could be implemented either in peer-to-peer between the primary and secondary BSs, or indirectly by the use of bridging terminals filling the gap between two separated neighboring cells.

\section{CONCLUSION}

In this paper, we have discussed the development of a radio enabler to assist reconfiguration management and ensuing technologies involving dynamic spectrum access. Such a radio enabler serves one of three building blocks defined in the IEEE P1900.4 Working Group, a standardization effort conceived to be a major link in the chain facilitating reconfiguration and resource optimization capabilities.

The detail presented on the radio enabler has highlighted its division into start-up and ongoing information provision phases, as well as some likely characteristics pertaining to the technical realization of these phases. Some technological constraints on the radio enabler have also been presented, as well as some specific use-cases for its operation involving network selection, optimization, and secondary spectrum usage.

\section{ACKNOWLEDGMENT}

This work has been performed within the project E2RII, which has received funding from the European Community's Sixth Framework Programme. This paper reflects only the authors' views; the Community is not liable for any use that may be made of the information contained herein. The contributions of colleagues from the E2RII consortium are hereby acknowledged.

\section{REFERENCES}

[1] IST-2005-027714-E²RII Deliverable D2.1, "Evolution of Reconfiguration Management and Control System Architecture," June 2006. Available from https://e2r2.motlabs.com/Deliverables

[2] Joseph Mitola III, "Cognitive Radio: An Integrated Agent Architecture for Software Defined Radio," PhD thesis, KTH Royal Institute of Technology, Stockholm, Sweden, 2000

[3] IEEE P1900.1 Standardisation Effort Homepage: http://grouper.ieee.org/groups/emc/emc/ieee_emcs_-_sdcom/P19001/ieee_emcs__p1900-1_main.htm

[4] P1900.4 Working Group Homepage: http:/grouper.ieee.org/groups/emc/emc/1900/4/index.htm

[5] IEEE 802.16 Working Group on Broadband Wireless Access Standards (WiMAX) Homepage: http://grouper.ieee.org/groups/802/16/

[6] IEEE 802.11 Working Group on Wireless Local Area Networks (WLANs) Homepage: http://grouper.ieee.org/groups/802/11/

[7] P. Cordier et al., "E2R Cognitive Pilot Channel Concept," IST 2006, Mykonos, Greece, June 2006

[8] P. Houze, S. Ben Jemaa, P. Cordier, "Common Pilot Channel for Network Selection," IEEE VTC 2006 Spring, Melbourne, Australia, June 2006 